\title{
Recognition Of A Chemical Casualty
}

\section{Introduction}

It is essential that medical personnel are familiar with the signs and symptoms of chemical agent poisoning to avoid repetition of the experience of World War I in which:

"medical officers frankly admitted that they were so handicapped by their lack of experience of cases of gas poisoning that they were often in doubt whether they were dealing with persons suffering from gas poisoning or not."

Medical and tactical intelligence channels should communicate with each other as early as possible. Threat information on potential use of CW weapons by enemy forces is important for the planning and execution of medical operations. Once CW weapons have been used, identification of agents and diagnosis will be an important element of medical intelligence for operational commanders.

\section{General}

Medical units should rely on information not only from detectors and intelligence sources but also from the individual casualties and their attendants. This applies particularly to agents for which, at present, there is no satisfactory detector - such as incapacitating agents. Some of the problems in the recognition and diagnosis of casualties suffering from the effects of chemical operations are discussed here. Medical personnel must bear in mind that with nerve agents, for example, symptoms and signs may range from mild, such as miosis, headache and tightness of the chest to signs and symptoms associated with severe poisoning, such as convulsions and respiratory failure. The nature and timing of symptoms will vary with the route of exposure. Although choking agents are less likely to be employed, the possibility of exposure to lung damaging agents should not be forgotten, and in particular it is essential that the quiescent period which follows the initial effects from poisoning is not mistaken for recovery and personnel sent back to duty, even after a lethal dose.

Battle casualties whose behavioural changes are not compatible with the physical signs of disability must be examined carefully to exclude the possibility of an incapacitant having been used. The possibility of unanticipated chemical hazards should not be discounted. When chemical agents have been released into the operational environment, it is important that the fullest and earliest information be given to medical units to facilitate the diagnosis of individual cases, the handling of casualties and their subsequent medical care.

\section{Recognition of a Chemical Casualty}

Any individual who unexpectedly becomes a casualty without being wounded or who is suffering a greater degree of incapacitation than is compatible with his or her wound should be considered a possible chemical casualty.

The differential diagnosis should consider the possibility of both direct or indirect effects of chemical exposures, with or without conventional injury. Indirect effects will include heat illness resulting from high levels of individual protection, psychological effects and those following the inappropriate use of autoinjectors for self and buddy aid. It is unlikely that a chemical attack or significant release of toxic chemicals would produce single casualties under field conditions; these exposures should be suspected with any sudden increase in numbers of unexplained casualties. If there is no threat from chemical weapons or evidence of a major release, and if only a few people are affected, another toxic hazard may be more probable (for example, carbon monoxide).

\section{Types of Casualties}

In the chemical environment, the following types of casualties may be seen:

1) Conventional Casualties. The conventional casualty with no chemical injury.

2) Direct Chemical Casualties.

- The chemical casualty with no other injury.

- The mixed casualty who has both a conventional and chemical injury. Since chemical munitions often include explosive burst charges, or may be delivered together with conventional munitions, such injuries may occur as part of a chemical agent attack. They may also occur when the chemical injury and conventional injury occur at different times. Other types of mixed casualties may occur if nuclear or biological weapons are used. Chemical injuries may occur combined with natural illness also. 
3) Indirect Chemical Casualties.

- Casualties suffering combat stress reaction (CSR). Combat stress reaction occurs often in warfare, but may be more frequent where a chemical warfare threat exists. The serviceman will have additional stresses arising from: (i) isolation as a result of wearing the chemical protective ensemble, (ii) additional fatigue from wearing the garments and (iii) fear of chemical agents. Experience in World War I showed that the differential diagnosis between the CSR casualties and chemical casualties may sometimes be difficult.

- Casualties with side effects from chemical agent antidotes. Some of the available antidotes may have undesirable side effects when taken inappropriately, or in large enough quantities. Atropine, for instance, causes decreased heat tolerance at a dose of $1 \mathrm{mg}$. Higher doses may cause tachycardia, dryness of the mouth, and decreased sweating. Medical personnel must be aware of the side effects of the available antidotes and be alert for their appearance.

- Heat casualty. Wearing the protective ensemble makes dissipation of excess body heat more difficult and increases heat stress. Wearing the respirator also complicates regular water intake. Both will increase the probability of heat exhaustion or heat stroke.

Management of casualties may be further complicated by the presence of chemical contamination on clothing and equipment.

\section{Questioning Casualties}

Under operational conditions, the medical situation may be complicated by the psychological impact on affected personnel exposed to chemical agents. The medical officer's questions should be along the following lines:

1) Determine whether the casualty has been caused by a chemical agent:

- Was the unit at high state of alert for chemical attack?

- Were there any aircraft or artillery bombardments in the area at the time of the attack?

- Was there any evidence of spray, liquid droplets or smoke?

- Was anybody else affected and if so, how was he or she affected?
- Did the casualty notice any unusual smell? (This is not a very reliable symptom under battle conditions, but it should be considered.)

- Did the available detection equipment respond positively?

- Did the casualty carry out any immediate action or decontamination drills?

2) Determine the identity of the agent:

- What subjective effects were noticed and how soon?

- An unexplained sudden runny nose.

- A feeling of choking or tightness in the chest or throat.

- Blurring of vision and difficulty in focusing the eyes on close objects.

- Any associated headache?

- Irritation of the eyes.

- Irritation of the skin.

- Unexplained difficulty in breathing or increased rate of breathing.

- Sudden feeling of depression.

- Anxiety or restlessness.

- Dizziness or light-headedness.

- Difficulty in concentration, confusion or disorientation.

- Slurred speech.

- Nausea or vomiting.

- Muscular weakness.

- Was there any delay between exposure or contamination and the onset of effects?

- Did the effects persist after adjustment or donning of the respirator?

- Has the casualty used any self-injection device? If so, did the symptoms improve or deteriorate?

- Is the casualty's behaviour normal?

3) Assess the dose of agent received:

- Was the casualty wearing full protective equipment at the time of the attack?

- Was the casualty in the open or under cover?

- Was the casualty exercising or at rest?

- Was there any delay in assuming full protection? For how long was the agent inhaled? How long was the interval between suspected contamination and decontamination?

- If there was a delay between exposure or contamination and the onset of effects, how long was this?

- Are signs and symptoms improving or deteriorating?

- Are there associated conventional injuries? 